\title{
SOLUBILITY ENHANCEMENT AND DISSOLUTION IMPROVEMENT- LIQISOLID TECHNIQUE
}

\author{
*Kapoor $D^{1}$, Sharma $S^{2}$, Patel $M^{1}$, Vyas RB ${ }^{1}$, Chaitali Lad ${ }^{1}$ \\ ${ }^{1}$ Dr. Dayaram Patel Pharmacy College, Sardarbaug, Station Road, Bardoli, Dist - Surat, Gujarat, India, Pin-394601 \\ ${ }^{2}$ Department of Pharmacy, BanasthaliVidyapith, Rajasthan, India, Pin-304022 \\ *Corresponding Author's E-mail id-dev7200@gmail.com, Contact Info - +91-7874223242
}

\begin{abstract}
:
This technique is based upon the admixture ofdrug loaded solutions with appropriate carrier and coating materials. The use of non-volatile solvent causes improvedwettability and ensures molecular dispersion of drug in the formulation and leads to enhance solubility. By usinghydrophobic carriers (non-volatile solvents) one can modify release (sustained release) of drugs by this technique.Liquisolid system is characterized by flow behavior, wettability, powder bed hydrophilicity, saturation solubility, drugcontent, differential scanning calorimetry, Fourier transform infra red spectroscopy, powder X-ray diffraction, scanningelectron microscopy, in-vitro release and in-vivo evaluation. The increased bioavailability is due to either increased surface area of drug available for release, an increased aqueous solubility of the drug, or improved wettability of the drug particles. According to the new formulationmethod of liqui-solid compacts, liquid medications such as solutions or suspensions of water insoluble drugs in suitable nonvolatileliquid vehicles can be converted into acceptably flowing and compressible powders by blending with selected powderexcipients. It has been speculated that such systems exhibit enhanced release profiles. In this case, even though the drug is in asolid dosage form, it is held within the powder substratin solution or, in a solubilized, almost molecularly dispersed state, whichcontributes to the enhanced drug dissolution properties. According to the new formulation method of liquisolid compacts, liquid medications suchas solutions or suspensions of water insoluble drugs in suitable non-volatile liquid vehicles can beconverted into acceptably flowing and compressible powders by blending with selected powder excipients. Large scale production of fabricated drug on commercial level successfulliquisolid tablet is a determination of optimal flowable liquid retention.
\end{abstract}

Keywords: Poorly soluble drugs, coating material, carrier, hydrophilic solvent, liquisolid compacts

\section{INTRODUCTION:}

The poorly soluble drug having dissolution rate too slow therefore uptake cannot be completed within the time at absorption site. If it remains in GIT for longer period may lead to decomposition of drug. There are two parameters useful for identifying poorly soluble drugs. One is its aqueous solubility should be less than $100 \mathrm{ug} / \mathrm{ml}$ and another is dose: solubility ratio. Dose: solubility ratio can be defined as volume of gastrointestinal fluids necessary to dissolve the administered dose. ${ }^{1}$

The liquisolid technique as described by Spireas is a novel concept, where a liquid may be transformed into a free flowing, readily compressible and apparently dry powder by simple physical blending with selected carrier and coating material. The liquid portion, which can be a liquid drug, a drug suspension or a drug solution in suitable nonvolatile liquid vehicles, is included into the porous carrier material. Inert, preferably water-miscible organic solvent systems with high boiling point such as liquid polyethylene glycols, propylene glycol, or glycerine are most excellent fitting as liquid vehicles. As the carrier is saturated with liquid, a liquid layer is formed on the particle surface which is instantlyadsorbed by the fine coating particles. $^{2}$

Solid dispersions consist of one or more active ingredients dispersed in a readily soluble solid hydrophilic matrix prepared by a melting (fusion) or solvent method ${ }^{3}$. With the melting method the drug is added to the molten carrier and the mixture is stirred until a homogenous melt is obtained. With the solvent method drug and carrier are dissolved in small amounts of solvent with final solvent evaporation. The higher release rates of solid dispersions may be ascribed to a number of factors which include formation of the amorphous form of the drug, reduction of particle size to nearly the molecular level, improved wetting properties and solubilisation of the drug by the carrier. The advantages of this methodology are the molecular dispersion of the drug within the hydrophilic carrier and the comparably high drug stability. However, for the preparation of solid dispersions usually special equipment is needed such as a spray dryer or a fluid bed apparatus. $^{4-9}$

\section{Liqisolid compact:}

Liquisolid technique is a new and promising method that can change the dissolution rate of drugs. It has been used to enhance dissolution rate of poorly water-soluble drugs. For poorly soluble (Class II) drugs and class (Class IV) the rate of oral absorption is often controlled by the dissolution rate in the gastrointestinal tract. The new "liquisolid", technique may be applied to formulate liquid medications (i.e., oily liquid drugs and solutions, suspensions or emulsions of water-insoluble solid drugs carried in nonvolatile liquid vehicles) into powders suitable for tableting or encapsulation. Since, the liquisolid tablets contain a solution of the drug in suitable solvent; the drug surface available for dissolution is tremendously increased. Due to significantly increased wetting properties and surface area of drug available for dissolution, liquisolid compacts of waterinsoluble substances may be expected to display enhanced drug release characteristics and, consequently, improved oral bioavailability. 
Drugs that can be incorporated into liquisolid systems:

Antihistaminic: chlorpheniramine

Antiarrthymic: digoxin, digitoxin

Antihypertensive: nifedipine

Antilipidemics: clofibrate, gemfibrozil

Antiepileptic: Carbamazepine, valproic acid.

Chemotherapeutic agent: etoposide.

Diuretics: Hydrochlorothiazide, methylchlorthiazide, polythiazide, spironolactone.

Glucocorticoids: prednisolone, hydrocortisone, prednisone.

NSAIDS: piroxicam, indomethacin, ibuprofen.

Water-insoluble vitamins: vitamin $\mathrm{A}, \mathrm{D}, \mathrm{E}$, and $\mathrm{K}$

\section{Advantages:}

- Huge number of Bio-Pharmaceutical classification class II drugs with high permeability, slightly or very slightly water soluble and practically insoluble liquids and solid drugs can be formulated into liquisolid systems.

- Improvement of bioavailability of an orallyadministered water insoluble drugs is achieved.

- This principle governs or administers the mechanismof drug delivery from liquisolid systems of powdereddrug solutions and it is mainly responsible for theimproved dissolution profiles exhibited by thispreparations.

- In this technique, production cost is low compared tosoft gelatin capsules.

- Drug is formulated in a tablet form or encapsulateddosage form and is held in solubilized liquid state, which confers developed or improved drug wettingproperties thereby improving drug dissolutionprofiles.

- Greater drug surface area is exposed to the dissolutionmedium. This liquisolid system is specifically for powderedliquid medications.

- Capability of industrial production is also possible.

- Enhanced bioavailability can be obtained as compared to conventional tablets.

- Differentiate the dosage form by admixture of colorinto liquid vehicle.

\section{Limitations ${ }^{10}$ :}

- Formulation of high dose lipophilic drugs theliquisolid tablet is one of the limitations of thistechnique.

- This techniques is not applicable forhigh dose insoluble drug.

- Mathematical calculations require.

\section{Advantages of liqisolid tablets over convention tablets:}

$\checkmark$ Liquisolid systems are low cost formulations than soft gelatin capsules.

$\checkmark$ Production of them is similar to that of conventional tablets.

$\checkmark$ Drug release can be modified using suitable formulation ingredients. $\checkmark$ Drug can be molecularly dispersed in the formulation.

$\checkmark$ Capability of industrial production is also possible.

$\checkmark$ Enhanced bioavailability can be obtained as compared to conventional tablets.

$\checkmark$ Omit the process approaches like nanonisation, micronization techniques.

$\checkmark$ Differentiate the dosage form by admixture of colour into liquid vehicle.

$\checkmark \quad$ To minimize excipients in formulation compare with other formulations like solid dispersions.

\section{Theory of liquid solid systems:}

A powder can retain only limited amounts of liquid whilemaintaining acceptable flow and compression properties. Tocalculate the required amounts of powder excipients (carrierand coating materials) a mathematical approach for theformulation of liqui-solid systems has been developed bySpirea,. This approach is based on the flowable ( $\Phi$-value) and compressible ( $\Psi$-number) liquid retentionpotential introducing constants for each powder/liquidcombination.

The $\Phi$-value of a powder represents the maximum amountof a given non-volatile liquid that can be retained inside itsbulk [w/w] while maintaining an acceptable flowability. Theflowability may be determined from the powder flow or bymeasurement of the angle of repose. The $\Psi$-number of apowder is defined as the maximum amount of liquid thepowder can retain inside its bulk [w/w] while maintainingacceptable compactability resulting in compacts of sufficienthardness with no liquid leaking out during compression. ${ }^{11,12,}$

Depending on the excipient ratio $(R)$ of the powder substratean acceptably flowing and compressible liquisolid systemcan be obtained only if a maximum liquid load on the carriermaterial is not exceeded. This liquid/carrier ratio is termed"liquid load factor $\mathrm{Lf}[\mathrm{w} / \mathrm{w}]$ and is defined as the weightratio of the liquid formulation $(W)$ and the carrier material $(Q)$ in the system:

$$
\mathrm{Lf}=\mathrm{W} / \mathrm{Q}------(1)
$$

' $R$ ' represents the ratio between the weights of the carrier $(Q)$ and the coating $(q)$ material present in the formulation:

$$
\mathrm{R}=\mathrm{Q} / \mathrm{q}------(2)
$$

The liquid load factor that ensures acceptable flowability(Lf ) can be determined by:

$$
\mathrm{Lf}=\Phi+\varphi \cdot(1 / \mathrm{R})-----(3)
$$

Where $\Phi$ and $\varphi$ are the $\Phi$-values of the carrier and coatingmaterial, respectively. Similarly, the liquid load factor forproduction of liqui-solid systems with acceptablecompactability $(\Psi L f)$ can be determined by:

$$
\Psi \mathrm{Lf}=\Psi+\psi \cdot(1 / \mathrm{R} \text {------- (4) }
$$

Where $\Psi$ and $\psi$ are the $\Psi$-numbers of the carrier and coatingmaterial, respectively.

\section{Classification:}


- Based on the type of liquidmedication contained therein, liquisolidsystems may be classified into threesubgroups:

$>$ Powdered drug solutions

$>$ Powdered drug suspensions

$>$ Powdered liquid drugs

The first two may be produced from theconversion of drug solutions or (e.g.prednisolone solution in propylene glycol) ordrug suspensions (e.g. gemfibrozilsuspension in Polysorbate 80), and thelatter from the formulation of liquid drugs(e.g. clofibrate, valproic acid, liquid vitamins,etc.), into liquisolid systems.

- Based on the formulation techniqueused, liquisolid systems may be classifiedinto two categories, namely,

$>$ Liquisolid compacts

$>$ Liquisolid microsystems

Liquisolid compacts are prepared using thepreviously outlined method to producetablets or capsules, whereas the liquisolidmicrosystems are based on a new conceptwhich to produce an acceptably flowingadmixture for encapsulations. $^{13}$

\section{Mechanism of enhanced drug release:}

\section{From liquid solid systems:}

Several mechanisms of enhanced drug release have beenpostulated for liqui-solid systems. The three main suggestedmechanisms include an increased surface area of drugavailable for release, an increased aqueous solubility of thedrug, and an improved wettability of the drug particles.

Formation of a complex between the drug and excipients orany changes in crystallinity of the drug could be ruled outusing DSC and XRPD measurements.

\section{a. Increased Drug Surface Area}

If the drug within the liqui-solid system is completelydissolved in the liquid vehicle it is located in the powdersubstrate still in a solubilized, molecularly dispersed state.Therefore, the surface area of drug available for release ismuch greater than that of drug particles within directlycompressed tablets ${ }^{12}$.

\section{b. Increased Aqueous Solubility of the Drug}

In addition to the first mechanism of drug releaseenhancement it is expected that $C s$, the solubility of the drug,might be increased with liqui-solid systems. In fact, therelatively small amount of liquid vehicle in a liquisolidcompact is not sufficient to increase the overall solubility ofthe drug in the aqueous dissolution medium. However, at thesolid/liquid interface between an individual liqui-solidprimary particle and the release medium it is possible that inthis microenvironment the amount of liquid vehiclediffusing out of a single liqui-solid particle together with thedrug molecules might be sufficient to increase the aqueoussolubility of the drug if the liquid vehicle acts as a cosolvent ${ }^{12}$.

\section{c. Improved Wetting Properties}

Due to the fact that the liquid vehicle can either act assurface active agent or has a low surface tension, wetting ofthe liqui-solid primary particles is improved.Wettability of these systems has been demonstrated bymeasurement of contact angles and water rising times. ${ }^{14}$

Many poorly soluble drugs have been formulated as liquisolidsystems showing enhanced drug release. Differentliquid vehicles, carrier and coating materials were used toformulate these drug delivery systems.

\section{Principle of Liquisolid Compacts:}

\section{Important terminologies in Principle:}

Liquid medication includes liquid lipophilic drugs and drug suspensions or solutions of solid water insoluble drugs in suitable non-volatile solvent systems.

Liquisolid system refers to powdered forms of liquid medications formulated by converting liquid lipophilic drugs, or drug suspensions or solutions of water insoluble solid drugs in suitable non-volatile solvent systems, into dry, nonadherent, free-flowing and readily compressible powder admixtures by blending with selected carrier and coating materials.

Carrier material refers to a preferably porous material possessing sufficient absorption properties, such as microcrystalline and amorphous cellulose, which contributes in liquid absorption.

Coating material refers to a material possessing fine and highly adsorptive particles, such as various types of silica, which contributes in covering the wet carrier particles and displaying a dry looking powder by adsorbing any excess liquid. $^{15}$

With the liquisolid technology, a liquid may be transformed into a free flowing, readily compressible and apparently dry powder by simple physical blending with selected excipients named the carrier and coating material. The liquid portion, which can be a liquid drug, a drug suspension or a drug solution in suitable non-volatile liquid vehicles, is incorporated into the porous carrier material (Fig. 1).

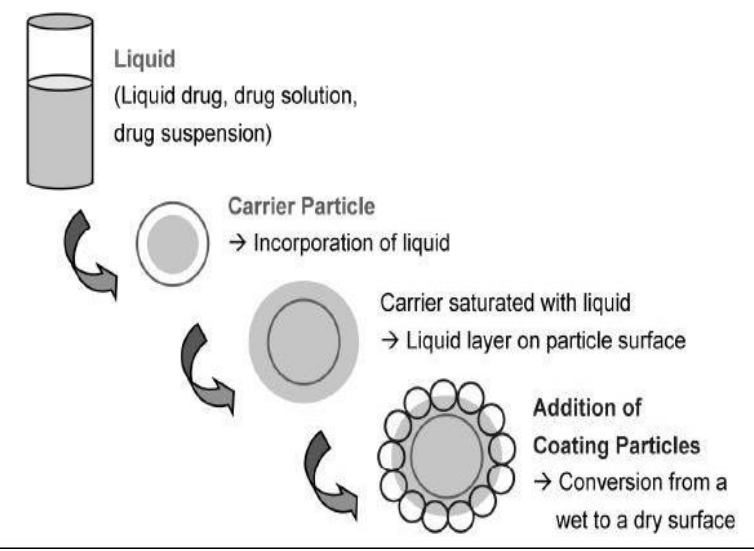

Figure 1: Schematic representation of liquisolid systems.

Inert, preferably water-miscible organic solvent systems with high boiling point such as propylene glycol, liquid polyethylene glycols, or glycerine are best suitable as liquid vehicles. Once the carrier is saturated with liquid, a liquid layer is formed on the particle surface which is instantly adsorbed by the fine coating particles. Thus, an apparently dry, free flowing, and compressible powder is obtained. Usually, microcrystalline cellulose is used as 
carrier material and amorphous silicon dioxide (colloidal silica) as coating material.

\section{Preparation of liquid solid compacts:}

As shown in figure, a liquid lipophilic drug(e.g.Chlorpheniramine, Clofibrate, etc.) can be convertedinto a liqui-solid system without being further modified. Onthe other hand, if a solid water-insoluble drug (e.g.Hydrochlorothiazide, Prednisone, etc.) is formulated, itshould be initially dissolved or suspended in a suitable nonvolatilesolvent system to produce a drug solution or drugsuspension of desired concentration. Next, a certain amount of the prepared drug solution orsuspension, or the liquid drug itself, is incorporated into aspecific quantity of carrier material which should bepreferably of a porous nature and possessing sufficientabsorption properties, such as powder and granular grades ofmicrocrystalline and amorphous cellulose are most preferredas carriers. The resulting wet mixture is then converted intoa dry-looking, non adherent, free-flowing and readilycompressible powder by the simple addition and mixing of acalculated amount of coating material. Excipients possessingfine and highly adsorptive particles, such as various types ofamorphous silicon dioxide (silica), are most suitable for thisstep. Before compression or encapsulation, variousadjuvants such as lubricants and disintegrates (immediate)or binders (sustained-release) may be mixed with thefinished liqui-solid systems to produce liqui-solid compactsi.e. tablets or capsules. ${ }^{16,17,18}$

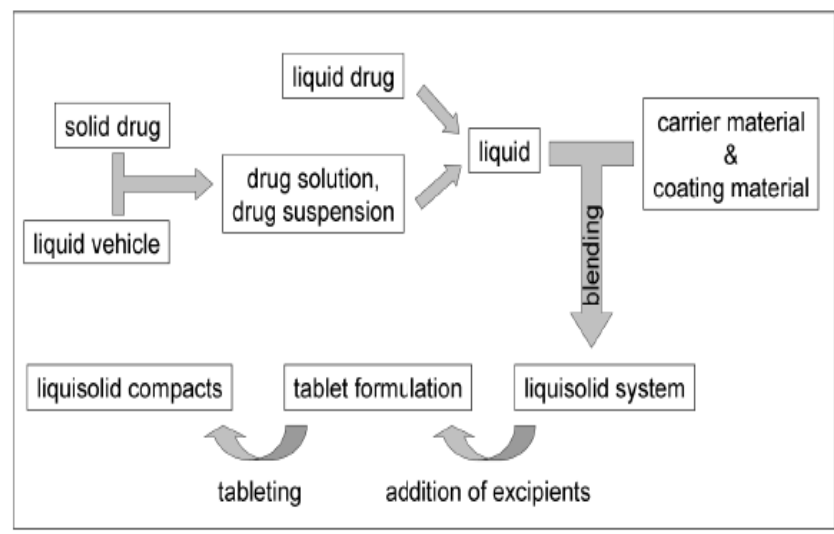

Figure 2: Schematic outline of the steps involved in the preparation of liquisolid compacts

Pre-compressionevaluation parameters of liquid solid systems:

Flow Properties of the Liqui-Solid System: ${ }^{19,20}$

The flow properties of the liqui-solid systems wereestimatedby determining the angle of repose, Carr'sindex, andHausner's ratio. The angle of reposewas measured by thefixed funnel and freestanding conemethod. The Bulk densityand Tap densities were determined for the calculation ofHausner's ratio andCarr's Index.

\section{Angle of repose:}

The angle of repose physical mixtures of liquisolidcompacts were determined by fixed funnel method. Theaccurately weighed physical mixtures of liquisolidcompacts were taken in a funnel. The height of the funnelwas adjusted in such a way that the tip of the funnel justtouches the apex of the heap of the powder. The powder wasallowed to flow through the funnel freely into the surface.The height and diameter of the powder cone was measuredand angle of repose was calculated.

\section{$\operatorname{Tan} \theta=\mathbf{h} / \mathbf{r}$}

Where, $\theta$ is the angle of repose, $\mathrm{h}$ is the height in cms, $\mathrm{r}$ is the radius in $\mathrm{cms}$

Values for angle of repose $\leq 300$ usually indicate a freeflowing material and angles $\geq 400$ suggest a poorly flowingmaterial. 25- 30 showing excellent flow properties,31-35showing good flow properties, 36-40 showing fair flowproperties, 41-45 showing passable flow properties.

\section{Bulk Density:}

The loose bulk density and tapped density were determinedby using bulk density apparatus. Apparent bulk density wasdetermined by pouring the blend into a graduated cylinder.The bulk volume $(\mathrm{Vb})$ and weight of the powder (M) wasdetermined. The bulk density was calculated using theformula:

\section{$\mathrm{Db}=\mathrm{M} / \mathrm{Vb}$}

where, $\mathrm{M}$ is the mass of powder, $\mathrm{Vb}$ is bulk volume of powder

\section{Tapped Density:}

The measuring cylinder containing a known mass of blendwas tapped for a fixed time. The minimum volume (Vt)occupied in the cylinder and the weight $(\mathrm{M})$ of the blendwas measured. The tapped density was calculated using theformula:

\section{$\mathbf{D t}=\mathbf{M} / \mathbf{V t}$}

Where, $\mathrm{M}$ is the mass of powder, $\mathrm{Vt}$ is tapped volume of powder

\section{Carr's Index (\%):}

The compressibility index has been proposed as an indirectmeasure of bulk density, size and shape, surface area,moisture content and cohesiveness of material because all ofthese ca influence the observed compressibility index. The simplest way for measurement of free flow of powder isCarr's Index, a indication of the ease with which a materialcan be induced to flow is given by Carr's index (CI) whichis calculated as follows:

$$
\begin{aligned}
& \text { CI }(\%)=[(\text { Tapped density }- \text { Bulk density }) / \\
& \text { Tappeddensity] x } 100
\end{aligned}
$$

The value below $15 \%$ indicates a powder with usually givesrise to good flow characteristics, where as above 25\% indicates poor flowability. 1-10 showing excellent flowproperties, 11-25 showing good flow properties 1620showing fair to passable, 21-25 showing passable.

\section{Hausner's Ratio:}

Hausner's ratio is an indirect index of ease of powder flow.It is calculated by the following formula.

\section{Hausner's Ratio=Tapped density ( $\rho t)$ / Bulk density ( $\rho b$ )}

Where $\rho t$ is tapped density and $\rho b$ is bulk density. LowerHausner's ratio $(<1.25)$ indicates better flow 
properties thanhigher ones, between 1.25 to 1.5 showing moderate flowproperties and more than 1.5 poor flow.

\section{Fourier Transform Infra Red Spectroscopy (FT-IR):}

FT-IR spectra of prepared melt granules are recorded on FTIR-8400 spectrophotometer. Potassium bromide (KBr) pellet method is employed and background spectrum is collected under identical situation. Each spectrum is derived from single average scans collected in the region $400-4000 \mathrm{~cm}-1$ at spectral resolution of $2 \mathrm{~cm}-2$ and ratio against background interfereogram. Spectra are analyzed by software. ${ }^{21}$

\section{Differential scanning calorimetry (DSC):}

Differential scanning calorimetry (DSC) is performed in order to assess the thermotropic properties and the thermal behaviors of the drug, excipients used in the formulation of the liquisolid system. Complete disappearance of characteristic peaks of drug indicates the formation of drug solution in the liquisolid powdered system, i.e., the drug is molecularly dispersed within the liquisolid matrix. ${ }^{21,22,23}$

\section{X-ray diffraction (XRD):}

For the characterization of crystalline state, X-ray diffraction (XRD) patterns are determined for physical mixture of drug and excipients used in formulation and for the prepared liquisolid compacts. $^{24}$ Absence of constructive specific peaks of the drug in the liquisolid compacts in X-ray diffractogram specify that drug has almost entirely converted from crystalline to amorphous or solubilized form. Such lack of crystallinity in the liquisolid system was understood to be as a result of drug solubilization in the liquid vehicle i.e., the drug has formed a solid solution within the carrier matrix. This amorphization or solubilization of drug in the liquisolid compacts it may contribute to the consequent improvement in the apparent solubility and enhancement of dissolution rate of the drug. ${ }^{25}$

\section{Post compression evaluation parameter of liquid solid compacts:}

\section{Weight Variation:}

Twenty tablets were randomly selected from each batch andindividually weighed. The average weight and standarddeviation three batches were calculated. It passes the test forweight variation test if not more than two of the individualtablet weights deviate from the average weight by more thanthe allowed percentage deviation and none deviate by morethan twice the percentage shown. It was calculated on anelectronic weighing balance.

\section{Thickness:}

The thickness of liqui-solid tablets was determined by usingDigital micrometer. Ten individual tablets from each batchwere used and the results averaged.

\section{Hardness:}

The hardness of the tablets was determined by usingMonsanto hardness tester. Five individual tablets from eachbatch were and results averaged.

Friability:
The friability values of the tablets were determined using aRoche-type friabilator. Accurately weighed six tablets wereplaced in Roche friabilator and rotated at $25 \mathrm{rpm}$ for 4 min.Percentage friability was calculated using the followingequation.

$$
\text { Friability }=([\mathrm{WO}-\mathrm{W}] / \mathrm{WO}) \times 100
$$

Where,

$\mathrm{WO}=$ Weight of the tablet at time zero before revolution, $\mathrm{W}=$ Weight of the tablet after 100 revolutions.

\section{Disintegration Test}

Six tablets were taken randomly from each batch and placedin USP disintegration apparatus baskets Apparatus was runfor 10 minutes and the basket was lift from the fluid,observe whether all of the tablets have disintegrated.

\section{Contact angle measurement:}

For assessment of wettability, contact angle of liquisolid tablets is measured according to the imaging method. The commonly used method is to measure contact angle directly for a drop of liquid resting on a plane surface of the solid, the so-called imaging method. A saturated solution of the drug in dissolution media is prepared and a drop of this solution is put on the surface of tablets. The contact angles are calculated by measuring the height and diameter of sphere drop on the tablet. ${ }^{24}$

\section{Scanning electron microscopy (SEM):}

Scanning electron microscopy (SEM) is utilized to assess the morphological characteristics of the raw materials and the drug-carrier systems. ${ }^{25}$

\section{In-vitro dissolution studies:}

Works of many researchers revealed that technique of liquisolid compacts could be a promising alternative for formulation of water-insoluble drugs. This technique of liquisolid compacts has been successfully employed to improve the in-vitro release of poorly water soluble drugs as hydrocortisone, ${ }^{26}$ Prednisolone ${ }^{27}$ Carbamazepine ${ }^{28}$ Piroxicam ${ }^{24},{ }^{29}$ Also several water insoluble drugs nifedipine, gemfibrozil, and ibuprofen, have shown higher bioavailability in rats as compared to their commercial counterparts.

\section{In-vivo evaluation of liquisolid systems:}

This liquisolid technology is a promising tool for the enhancement of drug release of poorly soluble drugs. The absorption characteristics of Hydroclorothiazideliquisolid compacts in comparison with commercial tablets were studied in beagle dogs. Significant differences in the area under the plasma concentration-time curve, the peak plasma concentration and the absolute bioavailability of the liquisolid and the commercial tablets were observed. However, for the mean residence time, the mean absorption time, and the rate of absorption no significant differences were found. The absolute bioavailability of the drug from liquisolid compacts was $15 \%$ higher than that from the commercial formulation. ${ }^{30}$

\section{Stability studies:}

Drug content was determined after the crystals were charged for accelerated stability studies according to $\mathrm{ICH}$ 
guidelines. Samples were taken and analysed for specified intervals.

\section{Bioavailability assessment for different active} pharmaceutical ingredients:

Bioavailability assessment is required for liquisolidtechnique. Because it was proved that enhancing the drugreleases from the dosage form by determination of in-vitrorelease studies. So, this parameter should establish fordetermination of the efficacy of the formulation. ${ }^{30}$

Atorvastatin calcium (ATR) is a BCS class II drug used as alipid lowering agent by acting as HMGCoAreductaseinhibitor. The prepared liquisolid compacts of ATR showedhigher release rates compared to the directly compressedtablets. The pharmacokinetic parameters of liquisolidcompacts of ATR, such as the AUC, tmax and Cmax showedthe better bioavailability compared with the conventionalformulation. ${ }^{31}$

Liquisolid Compacts of Aceclofenacorodispersible tablets were formulation and evaluated. The study is based on the effect of combined mixture of super disintegrants disintegrating action on drug release. Propylene glycol, PEG 400, Tween 80, microcrystalline cellulose were used as carrier. The liquisolid compacts with Sodium starch glycolate added intra granularly and Crosspovidone extra granularly showed highest dissolution rate. Orodispersibleliquisolid compacts prepared with Tween 80 enhance the dissolution rate of aceclofenac to a larger extent. $^{32}$

\section{Approaches to augment dissolution of active pharmaceutical ingredient release from its immediate release tablets:}

Liquisolid compacts confirmed significantly higher drugrelease rates, in different dissolution media, compared totablets prepared by the direct compression method. It wasalso observed that the drug dissolution rate from liquisolidtablets were independent of the volume of dissolutionmedium, in difference to the plain tablets which exposedeclining drug release patterns with decreasing dissolutionvolumes. $^{27}$

Cyclosporine (CS) Self Micro-Emulsifying Tablet (SME), the tablets were prepared by the liquisolid compactiontechnique. Formulation consists of oil, surfactant and cosurfactantwhich were selected on the basis of solubility andemulsification ability for the SME formulation. In this study, the mixture of Lauroglycol FCC: Maisine 35-1 (1: $1 \mathrm{w} / \mathrm{w})$ was selected as the oil phase, PEG35 Castor Oil wasselected as the surfactant and PEG-400 was selected as theco-surfactant. 1 to 6 was selected as the ratio between thedrug and the mixture. An Emulsion could not be formed inseveral oils, such as Carpryol 90, Lauroglyol 90 andLauroglycol FCC even in which the CS has good solubility.Due to the cyclic structure of CS-A, some excipientsabsorbed the drug and could not be selected as carriermaterial and coating material, e.g., silica powders. Theliquisolid tablets were effective in enhancing dissolution ofCS-A, a poorly water-soluble drug. The tablets exhibitedgood flowability and compactability. The results showed thatthe liquisolid compaction technique is a promising alternativetechnique to improve the solubility and the dissolution rate, for poorly water-soluble drugs CSA. ${ }^{33}$

Naproxen is a non-steroidal anti-inflammatory drug (NSAID)commonly used for the reduction of fever, pain andinflammation. Liquisolid compacts change the properties ofnaproxen particles by simply dispersing the drug particles ina non-volatile hydrophilic liquid vehicle, which increase thewetting properties of drug particles, and enhances thedissolution rate and shows improved bioavailability of thedrug. At present, naproxen is available commercially in high

dose tablets between 250 and $500 \mathrm{mg}$; the liquisolidformulations may help in reduction of the dose also. $^{34}$

\section{CONCLUSION:}

This technique is a potential substitute for formulation of water-insoluble/soluble drugs. The improved rate of drug dissolution from liquisolid tablets is almost certainly due to an amplify in wetting properties and surface area of drug particles obtainable for dissolution. Rapid disintegration ratesare experimentally compared to conventional tablets. Hence they show improved release rates and greater bioavailability. By this technique, sustained drug delivery systems were also be developed for the water soluble drugs in which hydrophobic nonvolatile solvents are used as vehicles Alteration of formulation by use of definite agent's source it control the release of drugs from the liquisolid tablets. 


\section{REFERNECES:}

1. Spireas S. Liquisolid System and method of preparing same. U.S Patent 6423339B1, 2002.

2. Merisko E. Liversidgenanocrystals: resolving pharmaceutical formulation issues associated with poorly soluble compounds in: J.J matty (Ed), Particles, Marcel Dekker, Orlando, 2002.

3. Jarowski CI, Rohera BD, Spireas S. Powdered solution technology: principles and mechanism. Pharm Res. 1992; 9: 1351-1358.

4. Barzegar JM, Javadzadeh Y, Nokhodchi A, Siahi-Shadbad MR. Enhancement of dissolution rate of piroxicam using liquisolid compacts. II Farmaco. 2005; 60: 361-365.

5. Nokhodchi A, Hentzschel CM, Leopord CS. Drug release from liquisolid system: speed it up, slow it down. Expert Opin Drug Del. 2011; 8: 191-205.

6. El-Houssieny BM, Wahman LF, Arafa NMS. Bioavailability and biological activity of liquisolid compact formula of repaglinide and its effect on glucose tolerance in rabbits. Bio Sci Trends.2010; 4: 17-24.

7. Khaled KA, Asiri YA, El-Sayed YM. In-vivo evaluation of hydrochlorothiazide liquisolid tablet in beagles dogs. Int J Pharm. 2001; 222: 1-6.

8. Naseem A, Olliff CJ, Martini LG, Lloyd AW. Effects of plasma irradiation on the wettability and dissolution of compacts of griseofulvin.Int J Pharm.2004; 269, 443-450.

9. Craig DQM. The mechanisms of drug release from solid dispersions in water-soluble polymers. Int. J. Pharm., 2002, 231: 131-144.

10. Javadzadeh $\mathrm{Y}$ and Nokhodchi A. Liquisolid technique for dissolution rate enhancement of a high dose water-insoluble drug (carbamazepine). Int J Pharm. 341:26-34.

11. Spireas, S. Liqui-solid systems and methods of preparing same. U.S. Patent 6423339B1 2002.

12. Spireas, S., Sadu, S.Enhancement of prednisolone dissolution properties using liqui- Solid compacts.Int. J.Pharm. 1998, 166: 177-188.

13. Nokhodchi A. The effect of type and concentration of vehicles on the dissolution rate of a poorly soluble drug (indomethacin) from liquisolid compacts. J pharm Sci. 8(1):18-25.

14. Yadav, V.B., Nighute, A.B., Yadav, A.V., Bhise, S.B. Aceclofenac size enlargement by non aqueous granulation with improved solubility and dissolution. Arch. Pharm. Sci.and Res. 2009, 1: 115-122.

15. Rajesh V, Areefulla S, Mallikarjun V, Solubility and Dissolution Enhancement: An overview. Journal of Pharmacy Research.2010,3,141- 145.

16. Barzegar-Jalali M, Dastmalchi S. Kinetic analysis of chlorpropamide dissolution from solid dispersions. Drug Dev. Ind. Pharm.,2007, 33: 63-70.

17. Valizadeh H, Zakeri-Milani P, Barzegar-Jalali M, Mohammadi G, Danesh- Bahreini MA, Adibkia K, Nokhodchi A. Preparation and characterization of solid dispersions of piroxicam with hydrophilic carriers. Drug Dev. Ind. Pharm., 2007, 33: 45-56.

18. Valizadeh H, Nokhodchi A, Qarakhani N, Zakeri-Milani P, Azarmi S, Hassanzadeh D, LoebenbergR.Physicochemical characterization of solid dispersions of indomethacin with PEG 6000, Myrj 52, lactose, sorbitol, dextrin and Eudragit ${ }^{\circledR}$ E100. Drug Dev. Ind. Pharm.,2004,30: 303-317.

19. Bhise SB, Nighute AB, Yadav AV, Yadav VB, Aceclofenac size enlargement by non aqueous granulation with improved solubility and dissolution. Arch Pharm Sci\& Res. 2009; 1:115122.

20. Craig DQM. Pharmaceutical applications of DSC. In: Craig DQM, Reading M (eds). Thermal analysis of pharmaceuticals. Boca Raton, USA, CRC Press, 2007, pp. 53-99.

21. Grover R, Spireas S, Wang T. Effect of powder substrate on the dissolution properties of Methchrothiazideliquisolid compacts. Drug DevInd Pharm. 1999; 25: 163-168.

22. Asnaashari S, Javadzadeh Y, Siahi MR., A. Nokhodchi, An investigation of physicochemical properties of piroxicamliquisolid compacts. Pharm Develop Tech. 2007; 12: 337-343.

23. Rakshit P, Ridhish P, Moinuddin S. Formulation and evaluation of liquisolid compacts of piroxicam. Ind drugs.2007; 44: 967972.

24. Martindale, The Complete Drug Reference, 6 Edn, The Pharmaceutical Press, London, 1999, pp. 937.

25. Naseem A, Olliff CJ, Martini LG, Lloyd AW. Effects of plasma irradiation on the wettability and dissolution of compacts of griseofulvin.Int J Pharm.2004; 269, 443-450.

26. Sadu S, Spireas S, Grover R. In vitro release evaluation of hydrocortisone liquisolid tablets.J Pharm Sci. 1998; 87:867-872.

27. Spiro S, Srinivas S. Enhancement of Prednisolone dissolution properties using liquisolid compacts. Int J Pharm. 1998; 166:177188.

28. Rakshit P, Ridhish P, Moinuddin S. Formulation and evaluation of liquisolid compacts of piroxicam. Ind drugs.2007; 44: 967972.

29. Tayel SA, Louis D, Soliman V. Improvement of dissolution properties of carbamazepine through application of the liquisolid tablet technique. Eur J Pharm Bio pharm. 2008; 69: 342-347

30. Khaled KA, Asiri YA, El-Sayed YM. In-vivo evaluation of hydrochlorothiazide liquisolid tablet in beagles dogs. Int J Pharm. 2001; 222: 1-6.

31. Gubbi SR, Jarag R. Formulation and characterization of Atorvastatin calcium liquisolid Compacts. Asian J Pharm Sci. 2010; 2:50-60.

32. El-say KM, Samy AH, Fetouh MI. Formulation and Evaluation of oral dispersible Liquisolid Compacts of Aceclofenac. Int J Pharm Sci Rev Res 2010; 3:135-142.

33. Zhao YQ, Zhou S, Potharaju H, Lou HM, Brunson E, Almoazen H, Johnson J. Development of a self micro-emulsifying tablet of Cyclosporine- A by the liquisolid compact technique. IJPSR, 2011; 2(9): 2299-2308.

34. Tiong N, Amal AE. Effects of liquisolid formulations on dissolution of Naproxen.Euro Journal of Pharm \&Biopharm.2009; 73: 373-384 